\title{
Impact of age and sex on sudden cardiovascular death following myocardial infarction
}

\author{
S Z Abildstrom, C Rask-Madsen, M M Ottesen, P K Andersen, S Rosthøi, \\ C Torp-Pedersen, L Køber, on behalf of the TRACE Study Group
}

Heart 2002;88:573-578

See end of article for authors' affiliations

Correspondence to: Dr S Z Abildstrom,

National Institute of Public Health, Svanemollevej 25, DK-2100 Copenhagen,

Denmark;

stabil@dadlnet.dk

Accepted 12 June 2002

\begin{abstract}
Objective: To evaluate and compare the risk of sudden cardiovascular death (SCD) and non-SCD after myocardial infarction (MI) associated with age and sex.

Design: Cohort study of patients admitted with an enzyme verified acute $\mathrm{Ml}$ and discharged alive. Patients were followed up for up to four years.

Patients: 5983 consecutive hospital survivors of acute MI were enrolled in the TRACE (trandolapril cardiac evaluation) registry from 1990-92. Four age groups were prespecified: < 56, 56-65, 66-75, and $\geqslant 76$ years.

Main outcome measures: SCD was defined as cardiovascular death within one hour of onset of symptoms.

Results: There were 536 SCD and 725 non-SCD. SCD mortality was $4.8 \%$ in the youngest and $15.7 \%$ in the oldest age groups. Non-SCD mortality was $3.5 \%$ and $25 \%$, respectively. The ratio of SCD to non-SCD mortality varied from 1.44 in the youngest ( $<56$ years) to 0.55 in the oldest patients ( $\geqslant 76$ years). Age significantly increased both SCD and non-SCD risk ( $p<0.0001)$, but the increase in nonSCD risk was $40 \%$ higher $(p<0.0001)$. Male sex was associated with increased risk of SCD independently of age (risk ratio 1.34, $\mathrm{p}<0.005$ ). However, the absolute three year probability of SCD among women older than 66 years exceeded $10 \%$.

Conclusions: Compared with non-SCD the risk of SCD is relatively highest in the younger age groups, but the absolute risk of SCD is much higher among the upper age groups than the younger. The risk of SCD was slightly lower in women but not enough to warrant a different treatment strategy.
\end{abstract}

$\mathrm{T}$ he majority of cases of sudden cardiovascular death (SCD) are caused by ventricular arrhythmias. ${ }^{12}$ One study of high risk patients with prior myocardial infarction (MI) has shown that sudden death in particular was reduced with an implantable cardioverter defibrillator. Hence, it is important to study in detail the prevalence of SCD in high risk populations where intervention with implantable defibrillators potentially has a high efficacy. The Framingham heart study ${ }^{4}$ found that the risk of sudden death increased with age in both men and women, but that the risk for women is only one quarter that of men of identical age. This was also the case among patients with established coronary heart disease ${ }^{4}$ Furthermore, it is the general belief that even if the risk of sudden death increases with age the risk of non-sudden death increases significantly more. ${ }^{5}$ However, this has not been tested in an unselected or contemporary population of patients with coronary heart disease. ${ }^{6}$ The majority of studies of risk of sudden death have been performed in survivors of myocardial infarct. Many of these have enrolled patient groups with an upper age limit of 70 years $^{7}$ and excluded important patient groups including those with diabetes or atrial fibrillation. ${ }^{8}$ Against this background we have performed a study of a large consecutive cohort of myocardial infarct survivors with emphasis on the importance of age and sex on the risk of SCD and non-SCD.

\section{METHODS}

\section{Patients}

The TRACE (trandolapril cardiac evaluation) registry comprises consecutive patients admitted with MI to any of 27 Danish coronary care units from June 1990 to July 1992. The registry was formed while screening patients for entry into the TRACE study, in which patients with left ventricular dysfunction following an acute MI were randomly assigned to treatment with trandolapril or placebo. All patients were entered in the registry irrespective of whether their treatment was randomised. All participating units had complete regional uptake and there was no age limit for admission to the coronary care units. A total of 6676 consecutive patients admitted alive with MI verified by increased cardiac enzyme concentrations were screened and enrolled in the registry. Patients were screened one to six days after admission and the screening procedure included an echocardiography, full medical history, and recording of all events during the hospital stay. A patient could have been screened more than once but in the present study we used only data from the first screening of each patient. For this study of risk of sudden and non-sudden death only events taking place after hospital discharge were considered.

The TRACE study and registry were approved by the National Board of Health, the Danish Data Protection Agency, and all local ethics committees in Denmark. Echocardiography was available for $96 \%$ of all the patients screened.

\section{Echocardiographic evaluation}

The echocardiogram was evaluated centrally using the wall motion index method..$^{10}$ The left ventricular ejection fraction

Abbreviations: AVID, antiarrhythmics versus implantable defibrillators; CAMI, Canadian assessment of myocardial infarction; $\mathrm{CASH}$, cardiac arrest study Hamburg; CAST, cardiac arrhythmia suppression trial; CIDS, Canadian implantable defibrillator study; HERS, heart and

oestrogen/progestin replacement study; MADIT, multicenter automatic defibrillator implantation trial; MI, myocardial infarction; MPIP, multicenter post-infarction program; SCD, sudden cardiac death; TRACE, trandolapril cardiac evaluation 
Table 1 Baseline characteristics of 5983 myocardial infarction survivors according to age group

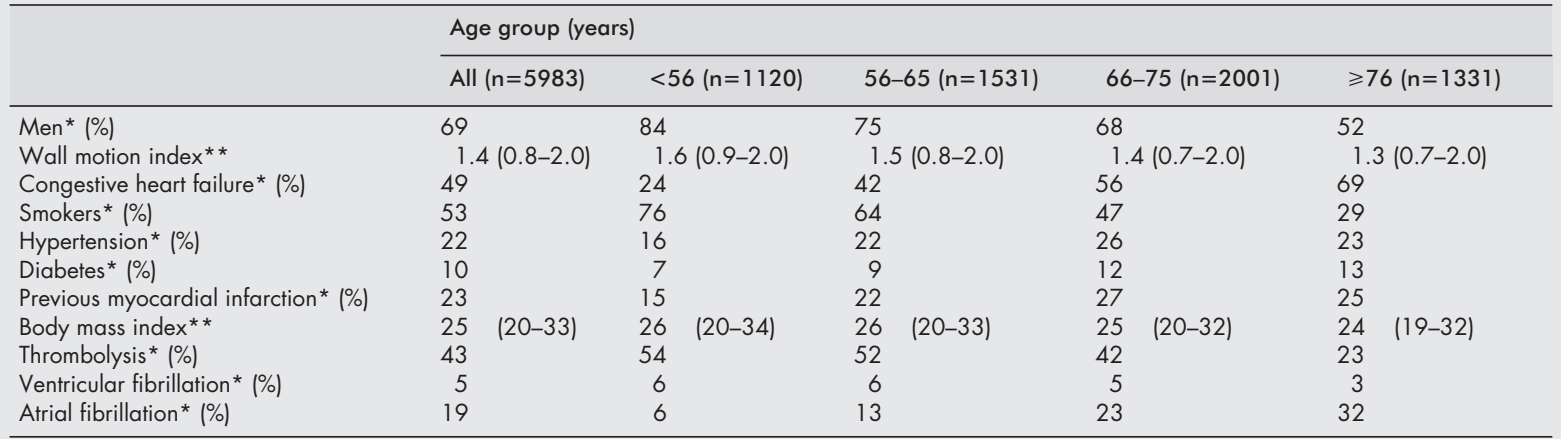

${ }^{*} p<0.001,{ }^{*} p<0.0001$ for no difference between the four age groups. Continuous variables are medians with 5 th and 95 th centiles. Discrete variables were compared by $\chi^{2}$ tests and continuous variables by rank sum tests.

as a percentage can be approximated by multiplying the wall motion index by 30 .

\section{Mortality}

Survival status among all patients screened was obtained two years after screening of the last patient on 15 July 1994 (median follow up 32 months). By making use of the Civil Registration System of Denmark we had to censor only 31 (28 non-Danish) patients at the time of hospital discharge, when they were last known to be alive.

\section{Sudden cardiovascular death}

SCD was defined as cardiovascular death within one hour of onset (or significant worsening) of symptoms leading to death. A central mortality committee classified all deaths with respect to cause and mode using information from death certificates, police investigations, hospital records, and necropsy reports when available. One committee classified death in the 1749 patients who had been randomly assigned to treatment in the TRACE study, and a separate committee used the identical criteria to classify those not randomly assigned to treatment. In 32 random cases both committees determined the mode of death. Differences in classification were found in five cases with a $\kappa$ statistic of 0.61 , which indicates good agreement.

Firstly, the committee classified whether death was caused by cardiovascular disease. Whenever the evidence was inadequate or uncertain the cause of death was classified as unknown. When information was available, death was assumed to be cardiovascular unless proved otherwise. Secondly, cardiovascular death was classified as sudden or non-sudden death with particular emphasis on the time elapsed from onset of new symptoms to death. Only cardiovascular death with a period documented to be less than one hour was classified as SCD. If there was any doubt concerning the elapsed time, the death was classified as nonSCD. Deaths of patients who died during their sleep, without any preceding symptoms and known to have been alive within 12 hours before their death, were classified as SCD. Deaths of patients admitted with cardiac arrest but who died after a period in coma following resuscitation were classified as SCD.

\section{Statistical methods}

The patient population was divided into four prespecified age groups: $<56,56-65,66-75$, and $\geqslant 76$ years. There were more than 1000 participants in each interval.

In univariate and multivariate studies of a single mode of death, Cox proportional hazard models were used. Variables with $\mathrm{p}<0.05$ in the models were considered to contribute independently. Since all patients in the analyses were required to have been discharged alive, and since lifetime was defined as time from MI to death or censoring, methods accounting for delayed entry (day of discharge) were applied.

The assumptions of the Cox model (possible interaction, and proportional hazards and log linearity of effects of quantitative covariates) were tested both graphically and numerically where appropriate.

The ratio between the risk of SCD and that of non-SCD associated with a given risk factor was estimated in a Cox model.

When evaluating other than all cause mortality, the particular mode of death was defined as the event while patients experiencing any other mode of death were censored. As the Kaplan-Meier estimator is not applicable to estimating probabilities of particular modes of death in a competing risk model, such probabilities were estimated as cumulative incidence functions. ${ }^{11}$ Cause specific hazards were statistically compared by the log rank test.

All analyses were performed using the SAS software (Statistical Analysis System, Cary, North Carolina, USA) version 6.12. The cumulative incidence functions were estimated from the output of the SAS PHREG procedure using a custom built program. The risk ratio denotes the hazard ratio.

\section{RESULTS}

Of the 6676 patients enrolled in the TRACE registry, 5983 were discharged alive from hospital and evaluated in this study. During follow up until June 1994, 1659 patients died. Among the deaths, 1261 were classified to be due to cardiovascular causes, 256 were classified to be due to non-cardiovascular causes, and 142 were unclassifiable because of inadequate information. Of the cardiovascular deaths 536 were classified as SCD and 725 as non-SCD.

The baseline characteristics differed between the four age groups (table 1). Table 2 shows the number of different modes of death in each age group.

The effect of increasing age on all modes of mortality was demonstrated to be log linear. There was no interaction between age and sex. Hazards were proportional for both age and sex.

The cumulative incidence of SCD in the entire population was $5.3 \%, 7.4 \%$, and $9.4 \%$ at one, two, and three years, respectively. The risk of both SCD and non-SCD increased significantly with increasing age (fig 1 ). The risk ratio associated with a 10 years increase in age (independently of sex) was 1.56 and 2.13 for SCD and non-SCD, respectively $(p<0.0001)$ (table 3$)$. The increase in non-SCD associated with increasing age was $40 \%$ higher than the increase in SCD risk $(\mathrm{p}<0.0001)$.

Table 4 gives the three year mortality from SCD and non-SCD in each age group. The ratio between risk of SCD and 
Table 2 Frequency of different modes of death according to age group

\begin{tabular}{lllll}
\hline \multicolumn{5}{c}{ Age group (years) } \\
\cline { 2 - 5 } & $\begin{array}{l}<6 \\
(n=1120)\end{array}$ & $\begin{array}{l}56-65 \\
(n=1531)\end{array}$ & $\begin{array}{l}66-75 \\
(n=2001)\end{array}$ & $\begin{array}{l}\geqslant 76 \\
(n=1331)\end{array}$ \\
\hline All cause death & 105 & 273 & 623 & 658 \\
Cardiovascular death & 79 & 209 & 477 & 496 \\
SCD & 46 & 109 & 205 & 176 \\
Non-SCD & 33 & 100 & 272 & 320 \\
Non-cardiovascular death & 15 & 39 & 97 & 105 \\
Unclassifiable death & 11 & 25 & 49 & 57 \\
\hline \multirow{2}{*}{$S C D$, sudden cardiovascular death. } & & & \\
\hline
\end{tabular}

risk of non-SCD decreased significantly with increasing age (table 4), from 1.44 in the youngest to 0.55 in the oldest $(\mathrm{p}<0.001)$.

In a multivariate analysis, male sex was associated with an increased risk of SCD and all cause mortality (table 3), but male sex had no predictive value concerning non-SCD independently of age (table 3). The increase in SCD risk associated with male sex was $28 \%$ higher than the increase in non-SCD risk $(\mathrm{p}=0.05)$.

Stratification of the analyses by sex showed that the age effect was similar in men and women, since no interaction was present. Figure 2 illustrates this, which shows the cause specific cumulative three year mortalities. Furthermore, fig 2 shows that the SCD risk of women lagged less than 10 years behind that of men.
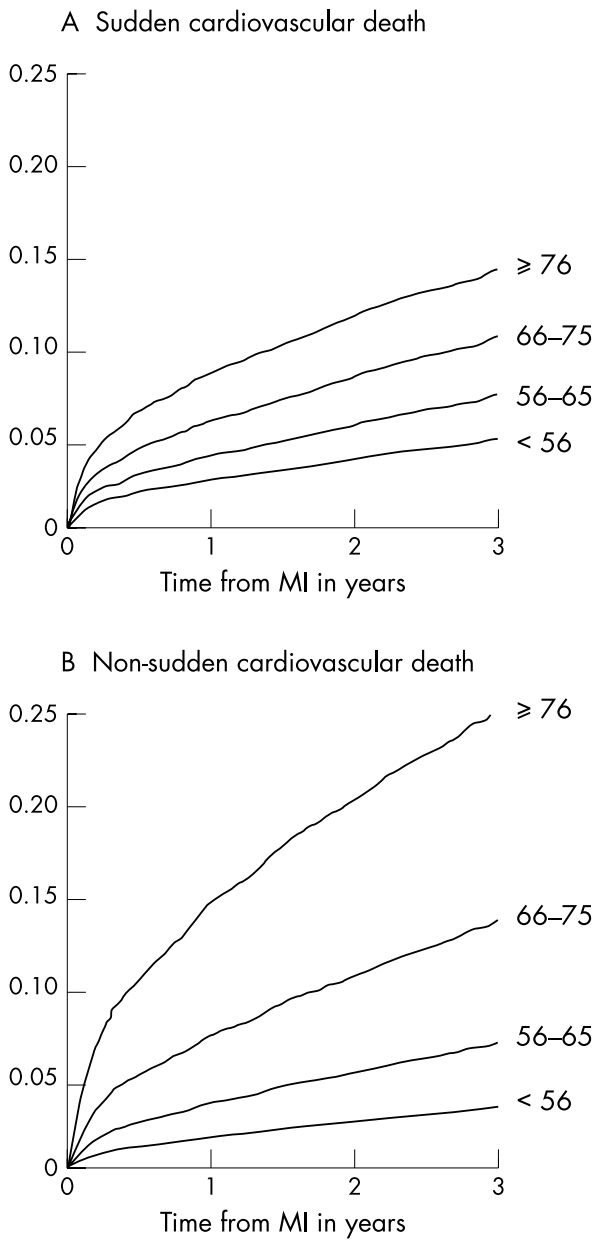

Figure 1 Mortality probability curves for sudden cardiovascular death and non-sudden cardiovascular death, according to age group.
Since the registry included patients both with and without randomly assigned treatment, the analyses were repeated with inclusion of information on randomisation and assigned treatment (trandolapril or placebo). Adjustment for these covariates did not affect the results regarding the impact of age and sex. The analysis of trandolapril versus placebo showed a similar effect of trandolapril treatment on SCD and non-SCD. Information about other medical treatment issued at discharge was not recorded and could not be analysed.

Adjustment for other risk factors likely to be associated with SCD or non-SCD risk and their relation did not change the results. Additional risk factors included in the model were wall motion index, congestive heart failure, New York Heart Association functional class, ventricular fibrillation, atrial fibrillation, smoking, body mass index, history of MI, hypertension, angina, or diabetes, and thrombolysis. The independent risk ratio associated with male sex was 1.25 $(p<0.05)$ for SCD and $1.12(p=0.2)$ for non-SCD. For a 10 year increase in age the risk ratios were 1.25 and 1.73 , respectively $(\mathrm{p}<0.0001$ for both SCD and non-SCD). Furthermore, decreased wall motion index, congestive heart failure, New York Heart Association functional class, atrial fibrillation, and history of hypertension, angina, or diabetes were all independently associated with increased risk of both SCD and non-SCD.

\section{DISCUSSION}

This is the first study of unselected and consecutive MI survivors to report the impact of age and sex on the risk of SCD and non-SCD. The principal findings of this study are that the risk of SCD increases with age, that the relation between risk of SCD and non-SCD decreases with age, and that male sex is associated with a slightly increased risk of SCD independent of age.

\section{Impact of age}

Our results are in perfect agreement with the results from the Framingham heart study, obtained from the general population during 38 years of follow up. ${ }^{4}$ Previous analyses of the subgroup with overt coronary heart disease ${ }^{12}$ did not show a clear effect of increasing age on the risk of SCD. ${ }^{13}$ The risk was found to be similar regardless of age for men. The proportion of SCD relative to all cardiovascular death was found to decrease, in a rather unstable fashion, with increasing age but conclusions were limited because of the small number of women. Before the introduction of thrombolysis, post-MI studies in Sweden $^{14}$ found a similar effect of age on the proportion of mortality being caused by SCD as we have reported here. The SCD rate was not given or tested in different age groups but seemed stable. No differences were found in the SCD risk between men and women. ${ }^{15}$ These studies included deaths during the initial hospitalisation, which may account for some of these differences.

In a study of 477 post-MI patients, Odemuyiwa and colleagues $^{8}$ divided the patients into two age groups (below and above 60 years) and found a similar SCD mortality in the 
Table 3 Multivariate Cox analysis of age, sex, and mode of death

\begin{tabular}{llll}
\hline & \multicolumn{2}{l}{ Risk ratio (95\% confidence interval) } \\
\cline { 2 - 4 } & All cause & SCD & Non-SCD \\
\hline Age per 10 years & $1.90(1.80-2.00)^{* * *}$ & $1.56(1.43-1.70)^{* * *}$ & $2.13(1.96-2.31)^{* * *}$ \\
Male sex & $1.18(1.06-1.31)^{* *}$ & $1.34(1.11-1.63)^{* *}$ & $1.05(0.90-1.23)^{*}$ \\
\hline${ }^{*} p>0.05 ;{ }^{* *} p<0.005 ;{ }^{* * *} p<0.0001$. & & \\
\hline
\end{tabular}

Table 4 SCD and non-SCD three year cumulative mortality probability

\begin{tabular}{lccl}
\hline Age (years) & SCD (\%) & Non-SCD (\%) & $\begin{array}{l}\text { Ratio between hazards } \\
\text { of SCD and non-SCD* }\end{array}$ \\
\hline$<56$ & 4.8 & 3.4 & 1.44 \\
$56-65$ & 7.3 & 6.9 & 1.09 \\
$66-75$ & 10.5 & 13.7 & 0.76 \\
$\geqslant 76$ & 14.2 & 25.3 & 0.55 \\
\hline
\end{tabular}

${ }^{*} p<0.001$ for no difference between age groups.

two groups: $4.7 \%$ and $3.2 \%$, respectively, and a significantly lower proportion of mortality from SCD in patients older than 60 years. In that study exclusion criteria were not having a Holter recording performed, age above 75 years, and any condition affecting heart rate variability other than ischaemic heart disease (that is, atrial fibrillation and diabetes mellitus). Owing to this selection the population studied had a relatively low mortality and the actual number of events was only 19 SCDs.

Many postinfarction studies have not classified death with respect to mode of death since their main purpose was to assess a change in all cause mortality between the different treatment groups. In the large $\beta$ blocker trials ${ }^{16-20}$ the mode of death was examined in the population randomly assigned to treatment, but the data published have specifically addressed the issue of whether $\beta$ blocker treatment reduces SCD. There have been no reports on the epidemiological distribution of SCD in these trials but, being part of randomised trials, patients are highly selected and not representative of the true postinfarction population.

\section{Impact of sex}

We found male sex to have an independent value in predicting SCD, while it had no influence on non-SCD risk. In the Framingham heart study male sex was found to increase the age adjusted SCD rate by a factor of four among patients with overt coronary heart disease. ${ }^{4}$ This may indicate that the absolute risk for women is so low that certain kinds of intervention would not be appropriate or cost effective. In contrast to this we found male sex to increase the risk of SCD by only 1.34 independently of age. This may result from differences between our population of MI survivors and the population with coronary heart disease in the Framingham heart study, defined as patients with angina pectoris and prior MI, including silent MI. An even more probable explanation may be the increase in female cardiovascular risk over time, which may be responsible for the observed difference, since the Framingham heart study reports results from 38 years of follow up. In the Framingham heart study the incidence of sudden death among women was similar to that among men 20 years younger. ${ }^{4}$ In our study the mortality caused by SCD among women in a given age group was always slightly higher than that of men in the younger age group. Thus, women lagged less than 10 years behind men with regard to the incidence of SCD.

To study closely the influence of menopause it would be tempting to divide the youngest group but, as is evident from table 2, the numbers of events are already low, especially when stratified by sex. The effect of sex on SCD risk was not statistically different in different age groups, as would be expected if hormone concentrations conferred a major risk increase, but a severe limitation in that aspect is the limited number of events among young women. Furthermore, women who have established coronary heart disease are more likely to be deficient in

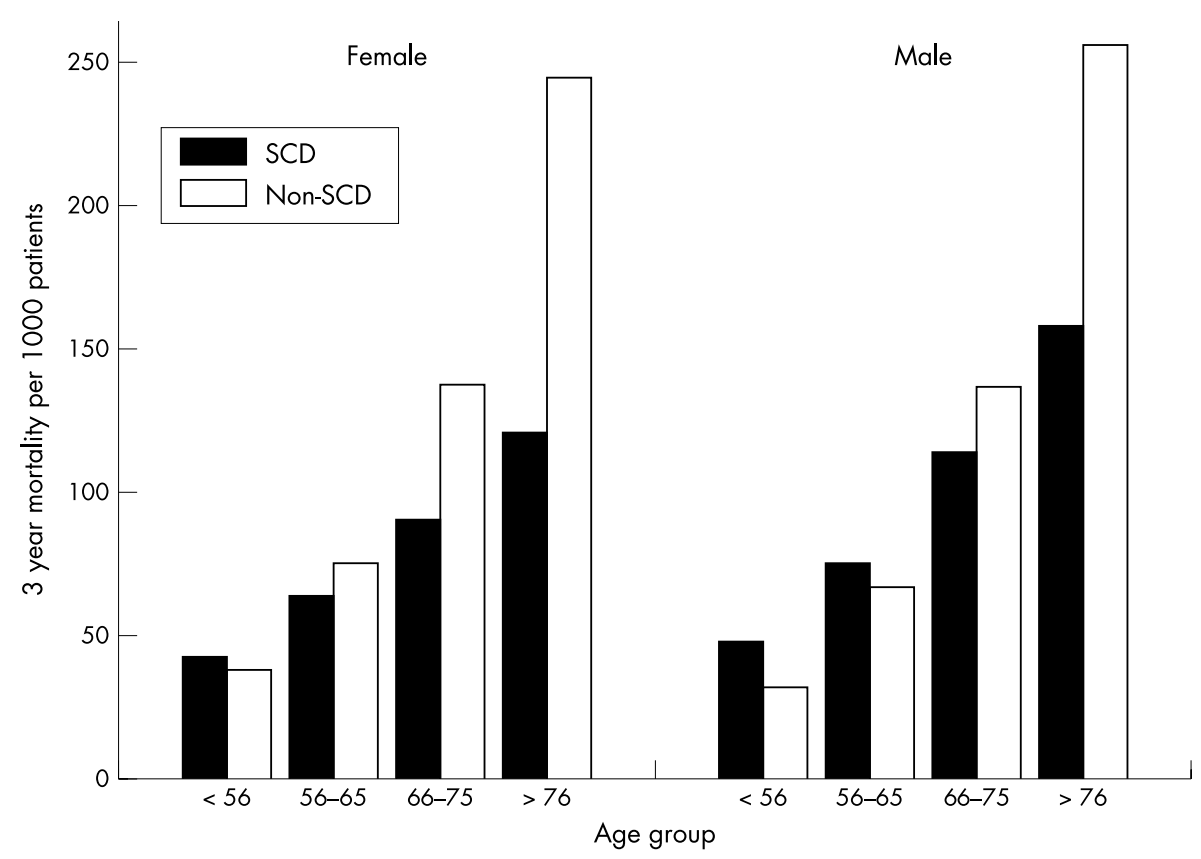

Figure 2 Cause specific three year mortality probability per 1000 patients for sudden cardiovascular death (SCD) and non-SCD according to age and sex. 
female sex hormones and this probably increases their cardiovascular risk. ${ }^{21}{ }^{22}$ Thus, the women in our study are highly selected compared with women in the general population, probably also with respect to hormone concentrations. The only reported randomised mortality study of hormone replacement therapy (HERS (heart and oestrogen/progestin replacement study) $)^{23}$ enrolled women with established coronary heart disease and no benefit was seen. Primary prevention trials are ongoing.

Male sex was weakly but significantly associated with an increased risk of all cause mortality, independently of age. In contrast, a recent study found that female sex adds risk independently of age following MI. ${ }^{24}$ This probably relies on the observation that female sex is associated with increased in-hospital mortality during acute MI. ${ }^{25}$ The present study included exclusively survivors of MI.

\section{The crude risk of sudden death}

The cumulative incidence of SCD during the first year was somewhat higher than in CAMI (Canadian assessment of myocardial infarction), ${ }^{26}$ another epidemiological study among post-MI survivors in the thrombolytic era. CAMI reported an incidence of arrhythmic death during the initial year following MI survival of only $1.9 \%$. The CAMI population was younger, since the initial 2477 patients were below 75 years of age. This is reflected in the all cause mortality rate (7.1\%), which is less than half that of our population (14.9\%) at one year. Furthermore, the CAMI study used the CAST (cardiac arrhythmia suppression trial) criteria $^{27}$ for classification of arrhythmic death, which tends to give a lower estimate than SCD. ${ }^{27}$ Comparison with the MPIP (multicenter post-infarction program $)^{7}$ is problematic since MPIP was conducted before thrombolysis in patients less than 70 years of age. Arrhythmic death was classified using the Hinkle and Thaler criteria.

\section{Pathophysiological considerations}

Age related pathoanatomical changes include diffuse myocardial fibrosis and myocyte disarray, which may provide an anatomical substrate for arrhythmia. ${ }^{28}$ Advanced age is also associated with changes in autonomic nervous system activity with increased sympathetic and decreased vagal tone, ${ }^{29} 30$ which may facilitate arrhythmia in the presence of an anatomical substrate. However, myocardial fibrosis and increased sympathetic activity may also be causal factors in the development of heart failure. Such mechanisms may explain the increased incidence in our study of both SCD and non-SCD death with increasing age. On the other hand, increased vagal activation may accompany acute myocardial ischaemia in women more often than in men, ${ }^{31}$ which may be one reason for male sex being a risk factor for SCD.

\section{Study limitations}

SCD may not always be arrhythmic. However, in several studies-MADIT (multicenter automatic defibrillator implantation trial), ${ }^{3}$ AVID (antiarrhythmics versus implantable defibrillators), CIDS (Canadian implantable defibrillator study), and CASH (cardiac arrest study Hamburg) ${ }^{32}$ - implantable cardioverter defibrillators have been found to reduce in particular death classified as arrhythmic, giving an indication that classification is indeed meaningful. The result of classification of mode of death is highly dependent on the amount of data available, ${ }^{33}$ but the data needed to classify death as SCD are usually available from the death certificate, emergency room charts, or hospital files. However, we are aware of the inaccuracy of death certificates and have avoided any default classification. This is reflected in the $8.6 \%$ of cases where information was inadequate to classify the mode of death.

We have no data on the medical treatment assigned at discharge. The increased use of $\beta$ blockers and statins is likely to reduce the absolute risk of both SCD and non-SCD. So far the effect of treatment has not been isolated to any particular age group or sex. This means that even if the absolute risk is reduced, the relative risk associated with age and sex remains the same despite changes in medical treatment. Similar considerations apply for the increased use of invasive treatments in post-MI patients.

\section{Implications}

The present study shows that, even if the proportion of cardiovascular mortality from SCD decreases with increasing age, the overall risk of SCD increases significantly. This implies that specific intervention against SCD should be tested or applied in all age groups of MI survivors. Furthermore, as the incidence of SCD is highest in the older age groups, the elderly will probably benefit the most from treatment as indicated by the CIDS trial. ${ }^{34}$ Effective treatment such as implantation of a cardioverter defibrillator should not be restricted to younger patients. Aggressive and effective treatment should be applied in the patients at highest risk, such as the elderly with a poor ejection fraction. The concept that these patients will die from other causes if SCD is prevented has never been found to be true.

Future interventional studies should include both men and women and investigate whether treatment effects are different between sexes. Since the crude SCD risk in men is only slightly higher than that in women, sex should not be considered when the indication for intervention is evaluated.

\section{ACKNOWLEDGEMENTS}

This study was in part supported by grants from Knoll Pharmaceutical Company, Ludwigshaven, Germany and from the Danish Heart Foundation (99-2-4-89-22769), Copenhagen, Denmark.

\section{Authors' affiliations}

S Z Abildstrom, National Institute of Public Health, Copenhagen, Denmark

C Rask-Madsen, M M Ottesen, C Torp-Pedersen, Department of Cardiology, Gentofte University Hospital, Copenhagen, Denmark

P K Andersen, S Rosthøi, Department of Biostatistics, Institute of Public Health, University of Copenhagen, Copenhagen, Denmark

L Køber, Department of Cardiology, Rigshospitalet, Copenhagen

University Hospital, Copenhagen, Denmark

\section{REFERENCES}

1 Hinkle LEJ, Thaler HT. Clinical classification of cardiac deaths. Circulation 1982;65:457-64.

2 Bayes de Luna A, Coumel P, Leclercq JF. Ambulatory sudden cardiac death: mechanisms of production of fatal arrhythmia on the basis of data from 157 cases. Am Heart J 1989;117:151-9.

3 Moss AJ, Hall WJ, Cannom DS, et al. Improved survival with an implanted defibrillator in patients with coronary disease at high risk for ventricular arrhythmia. Multicenter automatic defibrillator implantation trial investigators. N Engl J Med 1996;335: 1933-40.

4 Kannel WB, Wilson PW, D'Agostino RB, et al. Sudden coronary death in women. Am Heart J 1998;136:205-12.

5 Zipes DP, Wellens HJ. Sudden cardiac death. Circulation 1998;98:2334-51.

6 Priori SG, Aliot E, Blomstrom-Lundqvist C, et al. Task force on sudden cardiac death of the European Society of Cardiology. Eur Heart J 2001;22:1374-450.

7 Marcus FI, Cobb LA, Edwards JE, et al. Mechanism of death and prevalence of myocardial ischemic symptoms in the terminal event after acute myocardial infarction. Am J Cardiol 1988;61:8-15.

8 Odemuyiwa O, Farrell TG, Malik M, et al. Influence of age on the relation between heart rate variability, left ventricular ejection fraction, frequency of ventricular extrasystoles, and sudden death after myocardia infarction. Br Heart J 1992;67:387-91.

9 Køber L, Torp-Pedersen C, Carlsen JE, et al. A clinical trial of the angiotensin-converting-enzyme inhibitor trandolapril in patients with left ventricular dysfunction after myocardial infarction. Trandolapril cardiac evaluation (TRACE) study group. N Engl J Med 1995;333:1670-6.

10 Køber L, Torp-Pedersen C, Carlsen J, et al. An echocardiographic method for selecting high risk patients shortly after acute myocardial infarction, for inclusion in multi-centre studies (as used in the TRACE study). Trandolapril cardiac evaluation. Eur Heart J 1994;15:1616-20.

11 Andersen PK, Borgan $\varnothing$, Gill RD, et al. Statistical models based on counting processes. New York: Springer-Verlag, 1993. 
12 Kannel WB, Doyle JT, McNamara PM, et al. Precursors of sudden coronary death: factors related to the incidence of sudden death. Circulation 1975;51:606-13

13 Kannel WB, Schatzkin A. Sudden death: lessons from subsets in population studies. J Am Coll Cardiol 1985:5:141B-9B.

14 Herlitz J, Karlson BW, Bang A, et al. Survival, mode of death, reinfarction and use of medication during a period of 5 years after acute myocardial infarction in different age groups. Cardiology 1996:87:529-36.

15 Herlitz J, Bang A, Hartford M, et al. Influence of gender on survival, mode of death, reinfarction, use of medication, and aspects of well being during a period of five years after onset of acute myocardial infarction. Clin Cardiol 1996;19:555-61.

16 Norwegian Multicenter Study Group. Timolol-induced reduction in mortality and reinfarction in patients surviving acute myocardial infarction. N Engl J Med 1981;304:801-7.

17 Anon. A randomized trial of propranolol in patients with acute myocardial infarction. I. Mortality results. JAMA 1982;247:1707-14.

18 Anon. European Infarction Study (E.I.S.). A secondary prevention study with slow release oxprenolol after myocardial infarction: morbidity and mortality. Eur Heart J 1984;5:189-202.

19 Anon. Reduction in mortality after myocardial infarction with long-term beta-adrenoceptor blockade: multicentre international study: supplementary report. BN 1977; ii:419-21

20 Olsson G, Wikstrand J, Warnold I, et al. Metoprolol-induced reduction in postinfarction mortality: pooled results from five double-blind randomized trials. Eur Heart J 1992;13:28-32.

21 Anon. Effects of estrogen or estrogen/progestin regimens on heart disease risk factors in postmenopausal women. The postmenopausal estrogen/progestin interventions (PEPI) trial. The Writing Group for the PEPI Trial. JAMA 1995;273:199-208.

22 Bush TL. Evidence for primary and secondary prevention of coronary artery disease in women taking oestrogen replacement therapy. Eur Heart J 1996;17(suppl D):9-14
23 Hulley S, Grady D, Bush T, et al. Randomized trial of estrogen plus progestin for secondary prevention of coronary heart disease in postmenopausal women. Heart and Estrogen/progestin Replacement Study (HERS) Research Group. JAMA 1998;280:605-13.

24 Vaccarino V, Parsons L, Every NR, et al. Sex-based differences in early mortality after myocardial infarction. National registry of myocardial infarction 2 participants. N Engl J Med 1999:341:217-25.

25 Køber L, Torp-Pedersen C, Ottesen M, et al. Influence of gender on shortand long-term mortality after acute myocardial infarction. TRACE study group. Am J Cardiol 1996;77:1052-6.

26 Rouleau JL, Talajic M, Sussex B, et al. Myocardial infarction patients in the 1990s: their risk factors, stratification and survival in Canada: the Canadian assessment of myocardial infarction (CAMI) study. J Am Coll Cardiol 1996;27:1119-27.

27 Anon. Effects of encainide, flecainide, imipramine and moricizine on ventricular arrhythmias during the year after acute myocardial infarction: the CAPS. The cardiac arrhythmia pilot study (CAPS) investigators. Am $J$ Cardiol 1988:61:501-9.

28 Peters NS, Wit AL. Myocardial architecture and ventricular arrhythmogenesis. Circulation 1998;97:1746-54.

29 Tsuji H, Venditti FJJ, Manders ES, et al. Determinants of heart rate variability. J Am Coll Cardiol 1996:28:1539-46.

30 Reardon M, Malik M. Changes in heart rate variability with age. Pacing Clin Electrophysiol 1996;19:1863-6.

31 Airaksinen KE, Ikaheimo $M$, Linnaluoto $M$, et al. Gender difference in autonomic and hemodynamic reactions to abrupt coronary occlusion. J Am Coll Cardiol 1998:31:301-6.

32 Connolly SJ, Hallstrom AP, Cappato R, et al. Meta-analysis of the implantable cardioverter defibrillator secondary prevention trials. Eur Heart J 2000;21:2071-8

33 Torp-Pedersen C, Køber L, Elming $\mathrm{H}$, et al. Classification of sudden and arrhythmic death. Pacing Clin Electrophysiol 1997;20:2545-52.

34 Sheldon RS, Connolly S, Krahn A, et al. Identification of patients most likely to benefit from implantable cardioverter-defibrillator therapy. Circulation 2000;101:1660-4.

\section{IMAGES IN CARDIOLOGY}

\section{Near-fatal arrhythmia caused by hyperkalaemia}

$\Delta$ 75 year old woman with end stage diabetic renal disease was found with bradycardia (15-20 beats per minute) and asystole. After cardiopulmonary resuscitation (CPR) and administration of atropine (2 mg) and ephedrine $(100 \mu \mathrm{g})$, her heart rhythm converted to a stable rhythm, and she

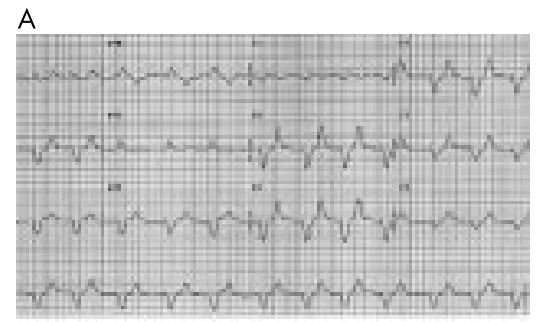

B

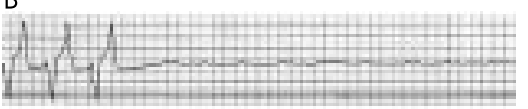

C

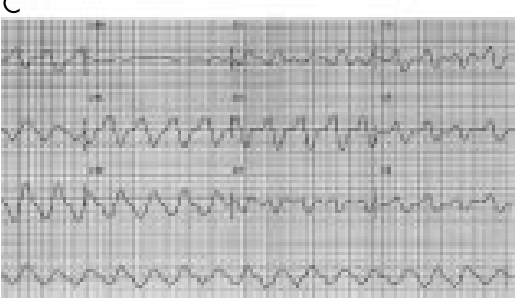

was transferred to the hospital. On admission, the ECG revealed a normal sinus rhythm with prolonged PQ interval, widening of QRS complexes, and peaked $\mathrm{T}$ waves (panel A). Forty minutes later, complete atrioventricular heart block developed with no ventricular escape rhythm (panel B) again necessitating CPR

D

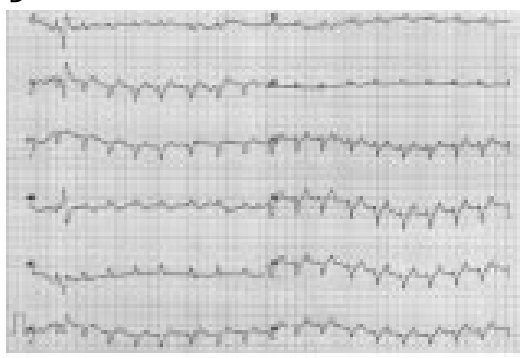

E

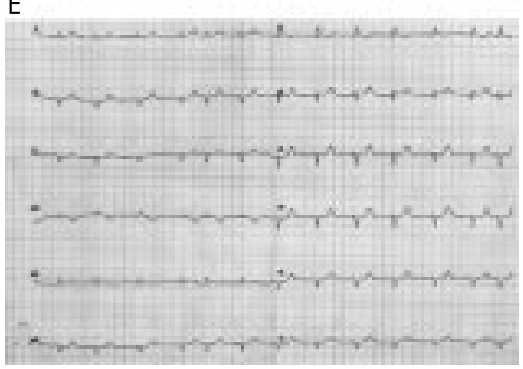

and administration of atropine and ephe drine. A subsequent ECG showed merging of QRS complexes with T waves (sine wave pattern) (panel C). At this time, the serum potassium concentration was $8.4 \mathrm{mmol} / \mathrm{l}$, and blood glucose was raised $(26.3 \mathrm{mmol} / \mathrm{l})$. The patient was treated with calcium gluconate, insulin, and sodium bicarbonate as well as immediate haemodialysis. Potassium lowering treatment resulted in progressive narrowing of QRS complexes (panel D, serum potassium concentration $7.1 \mathrm{mmol} / \mathrm{l}$; panel $\mathrm{E}$, serum potassium concentration $6.5 \mathrm{mmol} / \mathrm{l}$ ) within the next few hours. With a serum potassium of $4.4 \mathrm{mmol} / \mathrm{l}$, her ECG showed no changes to previous ECGs (panel F).

R B Walter

E B Bachli esther.baechli@dim.usz.ch

F

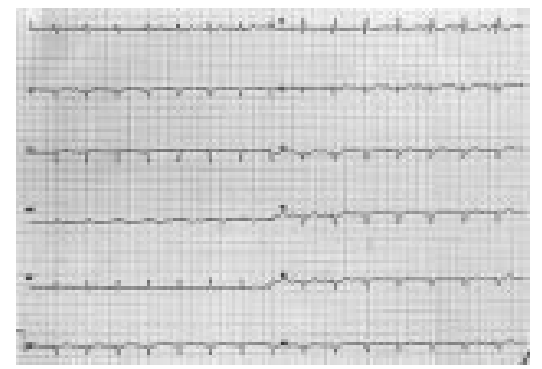

